FRI0565

\section{ERAMRS: A NEW MR SCORING SYSTEM FOR EARLY} RHEUMATOID ARTHRITIS

F. Xiao ${ }^{1}$, J. Yue ${ }^{2}$, L.-S. Tam², J. Griffith ${ }^{1} .{ }^{1}$ Dept of Imaging and Interventional Radiology; ${ }^{2}$ Dept of Rheumatology, The Chinese University of Hong Kong, Hong Kong, Hong Kong

Background: Early rheumatoid arthritis (ERA) is defined as having symptoms for less than 24 months and MRI allows quantification of several inflammatory features of ERA. Due to inclusion of some less relevant MR features and non-inclusion of other more relevant features, three MRI scoring systems currently employed are not ideally suited for use in ERA. We therefore devised a new more inclusive system suited for ERA, known as the ERA MR score ('ERAMRS'). The clinical relevance of this new ERAMRS system over the other MR scoring systems can be gauged by seeing how well these MR scoring systems correlate with clinical scoring systems.

Objectives: To (a) introduce a new scoring system (ERAMRS) for measuring the degree of inflammation on MR in early rheumatoid arthritis (RA) and (b) to see how well this ERAMRS system correlates with clinical scoring systems and serological parameters compared to currently used MR scoring systems.

Methods: 106 patients ( 81 females, 25 males, age, $53 \pm 12$ years) with early (i.e. symptoms $<24$ months) untreated RA underwent clinical/serological testing as well as $3 \mathrm{~T} \mathrm{MRI}$ of the most symptomatic wrist. Clinical assessment included Patient Global Assessment (PtGA), Physician Global Assessment (PGA) and Simple Disease Activity Index (SDAI). Erythrocyte sedimentation rate (ESR), and C-reactive protein (CRP) were also measured. ERAMRS (Early Rheumatoid Arthritis Magnetic Resonance Score) was semi-quantitatively assesses wrist joints synovitis ( $\mathrm{no}=0$, mild $=1$, moderate $=2$, severe $=3$ ) and enhancement (no=0, mild $=1$, moderate $=2$, severe $=3$ ), tenosynovitis (no=0, mild $=1$, moderate $=2$, severe $=3$ ) and enhancement $(\mathrm{no}=0$, mild $=1$, moderate $=2$, severe $=3$ ) as well as bone marrow oedema ( $\mathrm{no}=0,<50 \%$ of the whole bone $=1,>50 \%$ of the whole bone=2). Seven wrist joints, nine wrist tendons as well as 15 wrist bones were assessed. This ERAMRS method was compared to currently used methods of scoring inflammation on wrist MRI ('RAMRIS'[, 'McQueen'[, ${ }^{2}$ and 'MRI detected inflammation score' $\left[{ }^{3}\right)$.

Results: Synovitis was present in 104 (98\%), bone marrow oedema in 77 (73\%), and tenosynovitis in $82(77 \%)$ of the 106 wrists at presentation. RAMRIS system had the lowest correlation (PtGA, PGA, SDAI, CRP) or no correlation (ESR) while the ERAMRS system had the highest correlation with clinical disease activity level scoring systems $(r=0.44, p<0.001)$ and serological parameters $(r=0.41, p<0.001)$ among these four scoring systems.

Conclusions: The ERAMRS system, designed to grade inflammation on wrist MRI in early RA, provided the best correlation with clinical scoring systems and serological parameters indicating its improved clinical relevance over other MR scoring systems.

\section{REFERENCES:}

[1] Østergaard $\mathrm{M}$, et al. An introduction to the EULAR-OMERACT rheumatoid arthritis MRI reference image atlas. Ann. Rheum. Dis. 2005 Feb;64(suppl 1):i3-i7.

[2] Burgers LE, et al. Magnetic resonance imaging-detected inflammation is associated with functional disability in early arthritis - results of a crosssectional study. Rheumatology 2016 Dec;55(12):2167-2175.

[3] McQueen FM, et al. Magnetic resonance imaging of the wrist in early rheumatoid arthritis reveals a high prevalence of erosions at four months after symptom onset. Ann. Rheum. Dis. 1998 Jun;57(6):350-356.

Acknowledgements: NA

Disclosure of Interest: None declared

DOI: 10.1136/annrheumdis-2018-eular.6114

\section{FRI0566 OEDEMA-FIBROSIS IN SYSTEMIC SCLEROSIS: COMPARISON OF A PARAMETRIC CARDIOVASCULAR MAGNETIC RESONANCE MODEL TO THE LAKE LOUISE CRITERIA}

G. Markousis-Mavrogenis ${ }^{1}$, L. Koutsogeorgopoulou ${ }^{2}$, D. Apostolou ${ }^{3}$, G. Katsifis ${ }^{4}$, P. Argyriou ${ }^{3}$, S. Velitsista ${ }^{3}$, L. Papa ${ }^{3}$, M. Kanoupaki ${ }^{3}$, E. Agelopoulos ${ }^{3}$, E. Vernardos ${ }^{3}$, G. Kanoupakis ${ }^{3}$, G. Kolovou ${ }^{1}$, S.I. Mavrogeni ${ }^{1}{ }^{1}$ Cardiology Department, Onassis Cardiac Center, ${ }^{2}$ Pathophysiology Department, University Of Athens, Laiko Hospital; ${ }^{3}$ Cardiovascular Mri Unit, Mediterraneo Hospital; ${ }^{4}$ Navy Hospital, Athens, Greece

Background: Myocardial disease is a major cause of death in systemic sclerosis (SSc). We hypothesised that the in SSc patients with suspected myocarditis, the Lake Louise criteria underdiagnose myocarditis compared to diagnostic models that incorporate parametric cardiovascular magnetic resonance (CMR) indices (extracellular volume fraction [ECV], native T1- and T2-mapping).
Methods: 32 patients with diffuse SSc (dSSc) and a clinical suspicion of myocarditis were prospectively evaluated with a $1.5 \mathrm{~T}$ scanner using the Lake Louise criteria, and the aforementioned parametric indices were determined. The Lake Louise criteria were compared with parametric indices individually and in two models (with and without taking late gadolinium enhancement [LGE] into account), with regard to diagnostic agreement.

Results: Native T1-mapping and ECV individually had the greatest discordance with the Lake Louise criteria. The presence or absence of LGE in the proposed parametric models did not lead to changes in identified patient proportions. Native T1-mapping led to the same proportion of diagnosis of myocarditis as either parametric model, with the exception of 2 patients. Most importantly, patients identified only by parametric models, had a significantly subtler presentation than those identified by the Lake Louise criteria, as exemplified by significantly lower T2 ratio, early gadolinium enhancement and T2-mapping values.

Conclusions: In dcSSc patients with a clinical suspicion of myocarditis, novel parametric models including native T1-mapping, T2-mapping and ECV, identified a significantly greater proportion of patients with myocarditis compared to those identified by the Lake Louise criteria, and native T1 mapping had the greatest utility in this cohort.

Disclosure of Interest: None declared

DOI: 10.1136/annrheumdis-2018-eular.2837

\section{FRI0567 THE DIAGNOSTIC VALUE OF AUTOANTIBODY ISOTYPES IN RHEUMATOID ARTHRITIS}

D. Sieghart ${ }^{1}$, A. Platzer ${ }^{1}$, P. Studenic ${ }^{1}$, F. Alasti ${ }^{1}$, M. Grundhuber ${ }^{2}$, S. Swiniarski ${ }^{2}$, S. Blueml ${ }^{1}, \mathrm{H}$. Haslacher ${ }^{3}$, J. Smolen ${ }^{1}$, G. Steiner ${ }^{1}$. ${ }^{1}$ Department of Internal Medicine III, Division of Rheumatology, Medical University of Vienna, Vienna, Austria; ${ }^{2}$ ImmunoDiagnostic Division, Thermo Fisher Scientific, Phadia GmbH, Freiburg, Germany; ${ }^{3}$ Department of Laboratory Medicine, MEDICAL UNIVERSITY OF VIENNA, Vienna, Austria

Background: Anti-citrullinated protein antibodies (ACPA) and rheumatoid factor $(R F)$ are the most specific diagnostic markers of rheumatoid arthritis (RA). These antibodies are predominantly of the IgG (ACPA) or IgM (RF) isotype. Other subtypes of both antibodies - such as $\lg A$ - and other autoantibodies like RA33 have been repeatedly reported but their diagnostic value has still not been fully elucidated.

Objectives: To investigate the diagnostic value of $\lg A$, $\lg G$ and $\lg M$ subtypes of RF, ACPA and RA33 antibodies in patients with RA and their potential predictive value regarding therapeutic response to methotrexate (MTX).

Methods: To determine the diagnostic specificity and sensitivity, sera from 290 RA patients (including 165 MTX starter), 261 disease controls and 100 healthy subjects were tested for the presence of $\lg A$, $\lg G$ and $\lg M$ isotypes of RF, ACPA and RA33 by EliA ${ }^{T M}$ (Thermo Fisher Scientific). Cut-offs for prototype anti-RA33 $(\lg A, \lg G$ and $\lg M)$ and the $\lg M-A C P A ~ E l i A^{T M}$ were calculated by Receiver Operating Characteristic (ROC) curve analysis against disease controls and healthy subjects. In addition, RF and ACPA had been routinely measured by nephelometry and the anti-CCP EliA ${ }^{\mathrm{TM}}$, respectively.

Results: The most specific antibodies were $\lg G$ and $\lg A-A C P A$ as well as IgG$R F$, closely followed by IgG- and IgA-RA33 while IgM isotypes were found to be less specific. However, IgM-RF was the most sensitive isotype $(65 \%)$ followed by $\operatorname{lgG}-\mathrm{ACPA}(59.5 \%)$ and $\operatorname{lgA}-\mathrm{RF}(50.7 \%)$. Other subtypes were less prevalent ranging from $35 \%(\operatorname{IgA}-\mathrm{ACPA})$ to $6 \%(\operatorname{IgA}-\mathrm{RA} 33)$. Concerning RA33 antibodies, 14 patients were positive for IgA- and 18 for IgG-RA33. Interestingly, the major RA33 subtype was IgM which was detected in 43 patients. However, in contrast to RF and ACPA the overlap between the RA33 isotypes was marginal. RA33 antibodies as well as IgA-RF and IgA-ACPA were found to increase the diagnostic sensitivity of serological testing since they were found also in $22 \%$ of seronegative patients. Moreover, analysing IgM-RF by $\mathrm{Eli}^{\mathrm{TM}}$ proved more sensitive than $\mathrm{RF}$ measured by nephelometry which further reduced the number of seronegative patients. Thus, additional antibodies were detected in $30 \%$ of the seronegative population and, importantly, most patients had several antibodies, in contrast to disease controls which generally showed only one antibody species. The majority of antibody positive RA patients was found to be triple positive for RF, ACPA and either IgA-RF or IgA-ACPA. Among the 64 RA33 positive patients 48 were also positive for IgA-RF and/or lgA-ACPA (figure 1).

Interestingly, we found high levels of IgM-RF (>124 IU/ml) to be associated with achieving a SDAI50 response to MTX (17 of 24 cases). Furthermore, also the presence of RA33 antibodies was associated with a MTX response as $50 \%$ of RA33 positive patients $(n=32)$ achieved a SDAI50 response compared to $34 \%$ in the RA33 negative population $(p=0.034)$. 


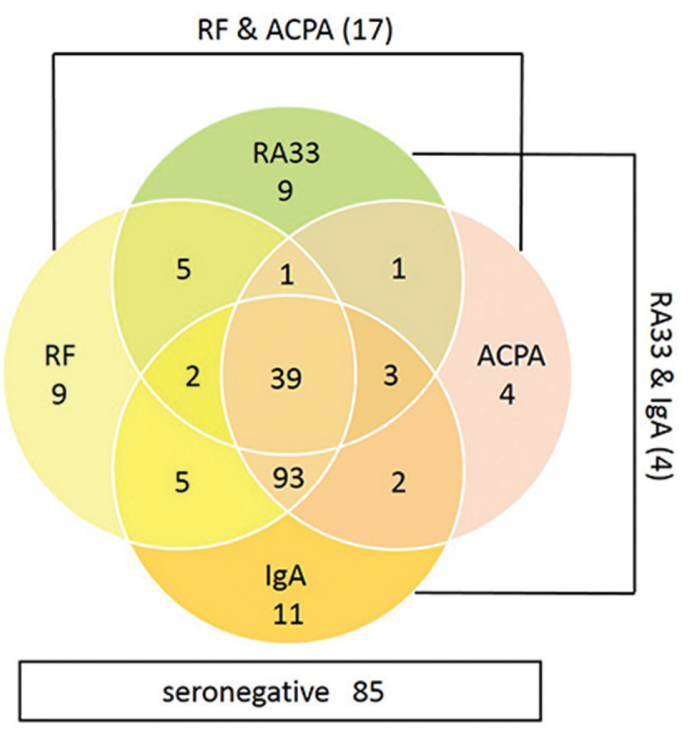

Abstract FRI0567 - Figure 1. Added diagnostic value of IgA-RF/ACPA and RA33 antibodies. Venn chart visualising the diagnostic overlap of RA33 and IgA antibodies with routine diagnostics (RF nephelometry, IgG-ACPA). The numbers of seronegative patients $(n=85), R F$ and IgG-ACPA positive but RA33 and IgA-RF/ACPA negative patients $(n=17)$ as well as patients positive for both RA33 and IgA-RF/ACPA but negative for RF and ACPA $(n=4)$ are also indicated

Conclusions: Thus, increasing the number of antibodies in serological routine testing would provide valuable additional information allowing to better distinguish between RA and other rheumatic disorders also in patients negative in current routine diagnostics and may provide valuable additional information regarding the prediction of treatment responses.

Disclosure of Interest: D. Sieghart: None declared, A. Platzer: None declared, P. Studenic: None declared, F. Alasti: None declared, M. Grundhuber Employee of: Thermo Fisher Scientific, Phadia GmbH, S. Swiniarski Employee of: Thermo Fisher Scientific, Phadia GmbH, S. Blueml: None declared, H. Haslacher: None declared, J. Smolen: None declared, G. Steiner: None declared DOI: 10.1136/annrheumdis-2018-eular.7532

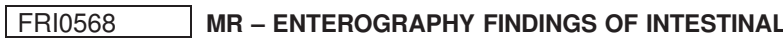 INFLAMMATION IN SPONDYLOARTHRITIS WITH HIGH FAECAL CALPROTECTIN LEVELS}

I. Ergenç ${ }^{1}$, E. Uner ${ }^{2}$, R. Ergelen ${ }^{3}$, G. Haklar ${ }^{2}$, N. İmeryüz $z^{4}$, Ö. Atuğ ${ }^{4}$, P. Atagündüz ${ }^{5} .{ }^{1}$ Internal Medicine; ${ }^{2}$ Biochemistry, ${ }^{3}$ Radiology; ${ }^{4}$ Gastroenterology, ${ }^{5}$ Rheumatology, Marmara University School of Medicine, Istanbul, Turkey

Background: Intestinal inflammation is seen in half of patients with spondyloarthropathy $(\mathrm{SpA})$, and can be detected by faecal calprotectin $(\mathrm{Fcal})^{1}$. Small bowel inflammation is detected by MR Enterography (MRE), accurately ${ }^{2}$.

Objectives: Definition of MRE changes in SpA patients with high Fcal levels.

Methods: A total of $231 \mathrm{SpA}$ patients, 50 healthy subjects and 50 inflammatory bowel disease (IBD) patients were included. Stool specimens were obtained and data on BASDAI scores, abdominal pain, diarrhoea, NSAID and TNF inhibitor usage were noted. Fcal was measured by IDK ELISA 2 weeks after the cessation of NSAID. MREs were obtained in Fcal $>50 \mu \mathrm{g} / \mathrm{g} \mathrm{SpA}$ patients. Contrast enhanced MRE was performed using 3 Tesla MRI device after 6 hours of fasting. Results: Demographic and clinical characteristics are summarised in table 1 and flow diagram (figure 1). Median Fcal of SpA patients was higher than healthy controls and lower than IBD patients. Fcal levels were high in 15 (33.3\%) of 45 healthy cases and all but two of them were NSAID users. The Fcal test was high in $45.9 \%$ $(n=106)$ of SpA patients. There was no correlation between BASDAI index and Fcal results $(r=0,08 p=0,42)$. Mean Fcal levels of active and in remission patients were $78.2 \pm 20$ and $77.8 \pm 7.02 \mu \mathrm{g} / \mathrm{g}$ respectively $(\mathrm{p}=0.97)$. Fifty-two of the Fcalhigh SpA patients were retested after cessation of NSAIDs for 14 days. In 15 patients Fcal levels remained elevated. MRE was performed $33 \mathrm{SpA}$ patients with high Fcal levels. MRE findings were observed in 29 of $33(87 \%)$ patients. Four out of $33(\% 12,1)$ MRE imaging was totally normal. Patients with MRE findings had higher median Fcal levels than those with normal MRE imaging (98 vs. $52 \mu \mathrm{g} / \mathrm{g}$ $\mathrm{p}=0.002$ ). All patients had increased mesenteric fat density and presence of mesenteric lymph nodes. Increased mesenteric vascularity (28/29), increased bowel wall enhancement (23/29) and thickness (18/29) were frequently detected.
Approximately one-third of the patients had a luminal stricture (13/29) and loss of haustration (12/13).

Abstract FRI0568 - Table 1. Demographic and clinical characteristics

\begin{tabular}{|c|c|c|c|c|}
\hline & $\begin{array}{c}\mathrm{SpA} \\
(\mathrm{n}=231)\end{array}$ & $\begin{array}{l}\text { Healthy } \\
\text { Controls } \\
(n=45)\end{array}$ & $\begin{array}{l}\text { IBD } \\
(n=50)\end{array}$ & $\begin{array}{l}\text { P Value (SpA vs } \\
\text { Healthy Cotrols) }\end{array}$ \\
\hline Age (Mean $\pm S D)$ & $45.2 \pm 9.8$ & $43.4 \pm 14.9$ & $\begin{array}{r}39.9 \\
\pm 13.1\end{array}$ & $\mathrm{p}=0.33$ \\
\hline Gender (Female/Male) & $117 / 114$ & $27 / 18$ & $18 / 32$ & $\mathrm{p}=0.25$ \\
\hline NSAID Users (\%) & $\% 54.8$ & \%53.3 & $\% 0$ & $\mathrm{p}=0.85$ \\
\hline NSAID dose/2 wks & $8.8 \pm 8.3$ & $10,6 \pm 8.3$ & 0 & $p=0.37$ \\
\hline Anti-TNF users (\%) & $\% 53.0$ & $\% 0$ & $\% 36.0$ & \\
\hline BASDAI (Mean $\pm S D)$ & $\begin{array}{c}2,58 \\
\pm 2,16\end{array}$ & & & \\
\hline $\operatorname{BASDAI} \geq 4(n / \%)$ & $\begin{array}{l}60 / 194 \\
(\% 26)\end{array}$ & & & \\
\hline Fcal (Median, Min-Max) & $\begin{array}{c}45.4(0- \\
830)\end{array}$ & $34.7(2-324)$ & $\begin{array}{c}69.5(0- \\
840)\end{array}$ & $p=0.004$ \\
\hline Fcal high $(>50 \mu \mathrm{g} / \mathrm{g})(\%)$ & $\begin{array}{c}\% 45.9 \\
(n=106)\end{array}$ & $\% 33.3(n=15)$ & $\begin{array}{l}\% 58.0 \\
(n=29)\end{array}$ & $\mathrm{p}=0.12$ \\
\hline $\begin{array}{l}\text { Fcal of NSAID Users } \\
\text { (Median, Min-Max) }\end{array}$ & $\begin{array}{c}63(0- \\
830)\end{array}$ & $54(2-324)$ & & $\mathrm{p}=0.30$ \\
\hline $\begin{array}{l}\text { Fcal of non-NSAID Users } \\
\text { (Median, Min-Max) }\end{array}$ & $\begin{array}{c}27(0- \\
507)\end{array}$ & $16(2-72)$ & & $\mathrm{p}=0.06$ \\
\hline
\end{tabular}

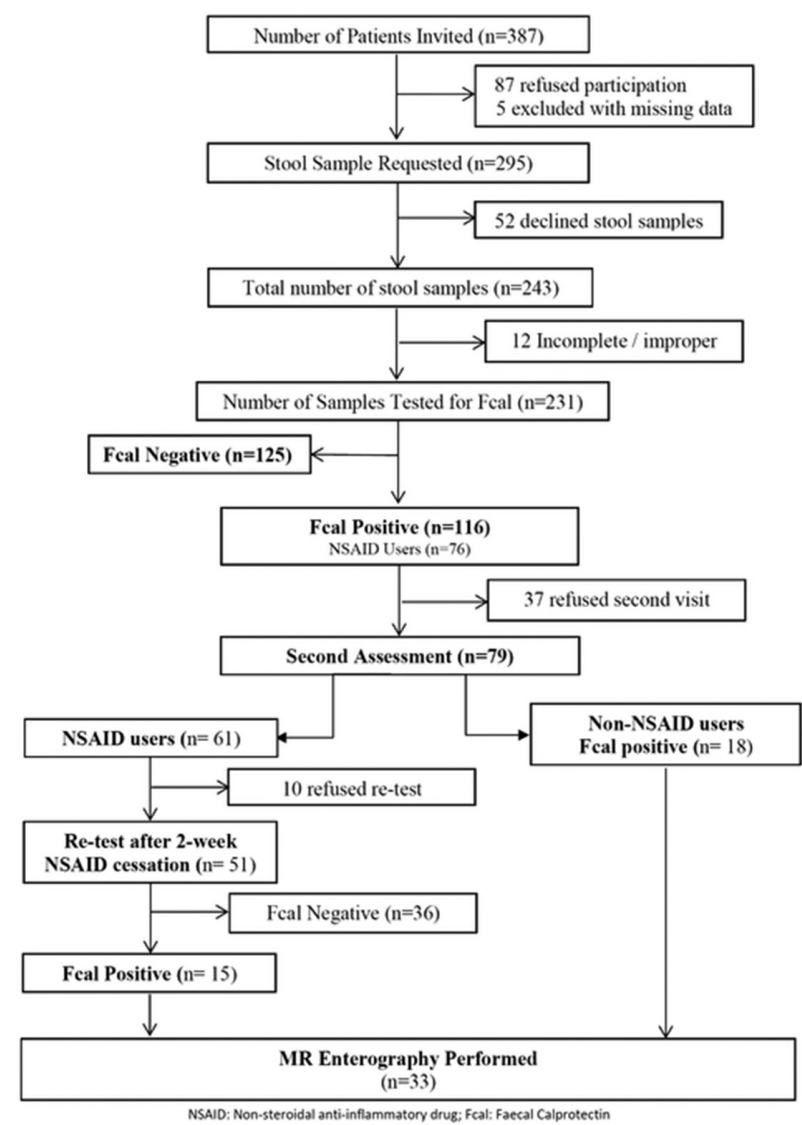

Conclusions: In SpA patients with high Fcal levels MRE detected inflammation of mucosal as well as serosal surfaces, as in early CD.

\section{REFERENCES}

[1] Cypers H. Elevated Calprotectin Levels Reveal Bowel Inflammation in Spondyloarthritis. ARD 75:1357-62.

[2] Toshimitsu Fujii. Current Progress of Endoscopy in Inflammatory Bowel Disease: Mr Enterography, Springer 2018:57-70.

Disclosure of Interest: None declared DOI: 10.1136/annrheumdis-2018-eular.3426 